\title{
ROBUST STABILIZATION OF NONLINEAR SYSTEMS WITH MARKOVIAN JUMPING PARAMETERS
}

\author{
E.K. Boukas \\ Mechanical Engineering Department \\ École Polytechnique de Montréal \\ P.O. Box 6079, station "Center-ville" \\ Montréal, Québec, Canada H3C 3A7. * \\ boukas@anas.meca.polymtl.ca \\ H. YANG \\ Department of Statistics \\ The University of Hong Kong \\ Hong Kong. ${ }^{\dagger}$ \\ hlyang@hkusua.hku.hk
}

\begin{abstract}
This paper deals with the class of uncertain nonlinear systems with Markovian jumping parameters. The uncertainties are assumed to be nonlinear and state dependent. The stabilization problem of this class of systems is studied here and sufficient conditions for the robust stabilizability are established.
\end{abstract}

\section{INTRODUCTION}

The class of systems with Markovian jumping parameters represents an interesting class of systems that we can use to model a variety of physical systems. This class of systems has two components in the state vector. The first one which varies continuously is referred to be the continuous state of the system

\footnotetext{
* Research of this author was supported by the Natural Sciences and Engineering Research Council of Canada under grants OGP0036444

${ }^{\dagger}$ Research of this author was supported by the Research grant C337/017/0019, Tne University of Hong Kong.
}

The original version of this chapter was revised: The copyright line was incorrect. This has been corrected. The Erratum to this chapter is available at DOI: 10.1007/978-0-387-35359-3_40 
and the second one which varies discretly is referred to be the mode of the system. This class of process was used in the past to model, for instance, manufacturing systems (see Sethi and Zhang (1994) or Gershwin (1994) and the references therein). Other applications using this class of systems can be found in Martion (1990) and the references therein.

In this paper, we will deal with the stochastic stabilizability and its robustness for the class of the nonlinear Markovian jumping parameters and try to establish conditions under which the optimal control of the nominal nonlinear system have a robust stabilizing control law for the uncertain nonlinear system. The uncertainty we will consider in this paper is a nonlinear and state dependent one. We will also consider the nonlinear changes in the control gain. The two nonlinearities will be assumed to be memoryless.

\section{PROBLEM STATEMENT}

Let us assume that the class of systems we consider in this paper be described by the following nonlinear differential equations:

$$
\begin{gathered}
\dot{x}(t)=A(x(t), r(t))+B(x(t), r(t))[d(x(t), r(t))+p(u(t))], \\
x(0)=x_{0}, r(0)=r_{0}
\end{gathered}
$$

where $x(t) \in R^{n}$ is the state vector of the system at time $t, u(t) \in R^{m}$ is the control input of the system at time $t(p(u(t))$ describes the nonlinear changes in the control gain) and $r(t)$ is a continuous-time Markov process taking values in a finite state space denoted by $\mathcal{S}=\left\{1,2, \ldots, n_{s}\right\} ; d(x(t), r(t)) \in R^{m}$ is the system disturbance; $A(x(t), r(t))$ and $B(x(t), r(t))$ are matrix functions with appropriate dimensions; $x(0)=x_{0}$ and $r(0)=r_{0}$ are respectively the initial values of the state and the mode at time $t=0$.

The evolution of the stochastic process $\{r(t), t \geq 0\}$ that determines the mode of the system is assumed to be described by the following probability transitions:

$$
P[r(t+h)=\beta \mid r(t)=\alpha]= \begin{cases}q_{\alpha \beta} h+o(h), & \text { if } \alpha \neq \beta \\ 1+q_{\alpha \alpha} h+o(h), & \text { otherwise }\end{cases}
$$

with $q_{\alpha \beta} \geq 0$ for all $\alpha \neq \beta$ and $q_{\alpha \alpha}=-\sum_{\beta \in \mathcal{S}} q_{\alpha \beta}$ for all $\alpha \in \mathcal{S}$, and $\lim _{h \rightarrow 0} \frac{o(h)}{h}=0$.

The control variable is assumed to be not constrained. Our objective in this paper is to seek an admissible feedback control law that minimises the following cost function:

$$
J_{u}\left(x_{0}, r_{0}\right)=\mathrm{E}\left[\int_{0}^{\infty}[g(x(t))+h(u(t))] d t \mid x(0)=x_{0}, r(0)=r_{0}\right]
$$

where $E$ stands for the mathematical expectation operator.

The question we will address in this paper is how can we design a control that stabilizes the system and guarantees its robustness. 
Let $x\left(t, x_{0}, \alpha\right)$ denote the trajectory of the state $x(t)$ from the initial state $\left(x_{0}, r_{0}\right)$. We introduce the following stochastic stability and stochastic stabilizability concepts for continuous-time jump nonlinear systems.

Definition 1 For system (2.1) with $u(t) \equiv 0$ (i.e. $p(u(t)) \equiv 0)$ and $d(x(t), r(t)) \equiv$ 0 , for all $r(t) \in \mathcal{S}$, the equilibrium point 0 is stochastically stable, if for every initial state $\left(x_{0}, r_{0}\right)$ the following:

$$
\int_{0}^{\infty} \mathrm{E}\left\{\left\|x\left(t, x_{0}, r_{0}\right)\right\|^{2}\right\} d t<\infty
$$

holds

Definition 2 We say that the system (2.1) is stochastically stabilizable, if for every initial state $\left(x_{0}, r(0)\right)$, there exists a feedback control law $u(t)=$ $-k(x(t), r(t))$, such that the closed-loop system

$$
\dot{x}(t)=A(x(t), r(t))+B(x(t), r(t))[d(x(t), r(t))+p(-k(r(t))]
$$

is stochastically stable for admissible uncertainty $d(x(t), r(t))$.

We will assume in this paper that the data of this optimization control problem are smooth enough to satisfy the existence of the control law of the nominal optimization control problem. The details of the assumptions will be given in the appropriate section. We will also assume that the mode of the system is completly accessible for control. The functions in the instantaneous cost will be chosen as follows:

$$
\begin{aligned}
& g(x(t), r(t))=g_{0}(x(t), r(t))+g_{1}(x(t), r(t)) \\
& h(x(t), r(t))=\frac{1}{2} u^{\top}(t) R(r(t)) u(t)
\end{aligned}
$$

where the function $g_{1}(x(t), r(t))$ is chosen to compensate the effect of the nonlinear state dependant disturbance $d(x(t), r(t))$ while the function $g_{0}(x(t), r(t))$ is chosen to accelerate the convergence rate under the worst possible disturbance $d(x(t), r(t))$. The expression of $h(u(t), r(t))$ is chosen to simplify the expression of the feedback control law of the nominal nonlinear optimization problem.

\section{JUMP NONLINEAR OPTIMIZATION PROBLEM}

To solve the optimization problem of the previous section, let us consider the more general case and then based on its solution get the one we are looking for. Let us consider that the system dynamics is described by the following differential nonlinear system of equations:

$$
\dot{x}(t)=f(x(t), u(t), r(t)), x(0)=x_{0}, r(0)=r_{0}
$$

where the variables above keep the same definitions as in previous section, with $f(0,0, \alpha)=0$ for all $\alpha \in \mathcal{S}$.

Let the cost function be defined by:

$$
J_{u}\left(x_{0}, r_{0}\right)=\mathrm{E}\left[\int_{0}^{\infty}[g(x(t), r(t))+h(u(t), r(t))] d t \mid x(0)=x_{0}, r(0)=r_{0}\right]
$$


where for each $\alpha \in \mathcal{S}$ we have $g(0, \alpha)=0$ and $g(x(t), \alpha)>0$ for all $x \neq 0$ for $g(x(t), r(t))$; and $h(0, \alpha)=0$ and $h(u(t), \alpha)>0$ for all $u \neq 0$ for $h(u(t), r(t))$.

Definition $3 \mathrm{~A}$ control $u(\cdot)=\{u(t): t \geq 0\}$ is said to be admissible if: (i) $u(\cdot)$ is adapted to the $\sigma$-algebra generated by the random process $r(\cdot)$, denoted as $\sigma\{r(s): 0 \leq s \leq t\}$, and (ii) $u(t) \in R$ for all $t \geq 0$.

Let $\mathcal{U}$ denote the set of all admissible controls of our control problem.

Definition 4 A measurable function $u(x(t), r(t))$ is an admissible feedback control, if (i) for any given initial $x$ and $\alpha$ of the continuous state and the mode, the following equations have an unique solution $x(\cdot)$ :

$$
\dot{x}(t)=f(x(t), u(t), r(t)), x(0)=x
$$

and (ii) $u(\cdot)=u(x(\cdot), r(\cdot)) \in \mathcal{U}$.

Let us assume that the functions $f(x(t), u(t), r(t)), g(x(t), r(t))$ and $h(u(t), r(t))$ satisfy all the required assumptions that guarantee the existence of the optimal feedback control law in the form:

$$
\begin{aligned}
& k: R^{n} \times \mathcal{S} \rightarrow R^{m} \\
& \quad(x(t), r(t)) \rightarrow k(x(t), r(t))
\end{aligned}
$$

Let the value function $v(x(t), r(t))$ be defined by:

$$
v(x(t), \alpha)=\min _{u(t)} J_{u}(x(t), \alpha) \text {, when } r(t)=\alpha
$$

Using the dynamic programming principle (see, Rishel (1975) or Boukas (1993)), and the expression of the value function, i.e.:

$$
v(x(t), \alpha)=\min _{u(t)} E\left[\int_{0}^{\infty}[g(x(s), r(s))+h(u(s), r(s))] d s \mid x(t), \alpha\right]
$$

it can be shown that the Hamilton-Jacobi-Bellman equation is given by the following:

$$
\min _{u(t)}\left[\left(\mathcal{A}_{u} v\right)(x(t), r(t))+g(x(t), r(t))+h(u(t), r(t))\right]=0
$$

where $\left(\mathcal{A}_{u} v\right)(x(t), r(t))$ is defined as follows:

$$
\begin{gathered}
\left(\mathcal{A}_{u} v\right)(x(t), r(t))=\quad v_{x}^{\top}(x(t), r(t)) f(x(t), u(t), r(t)) \\
+\sum_{\beta \in \mathcal{S}} q_{\alpha \beta} v(x(t), \beta)
\end{gathered}
$$

In the next section we will return to the problem of section 2 and try to find the optimal control law of the feedback type of the nominal nonlinear system Markovian jumping parameters. 


\section{OPTIMALITY CONDITIONS OF THE NOMINAL NONLINEAR SYSTEM}

The dynamics of the nominal nonlinear system of the one considered in section 2 is described by the following ordinary nonlinear differential equations:

$$
\dot{x}(t)=A(x(t), r(t))+B(x(t), r(t)) u(t), x(0)=x_{0}, r(0)=r_{0}
$$

with $A(0, \alpha)=0$ for all $\alpha \in \mathcal{S}$

If we choose the functions $g(x(t), r(t))$ and $h(u(t), r(t))$ of the instantaneous cost as follows:

$$
\begin{aligned}
& g(x(t), r(t))=g_{0}(x(t), r(t))+g_{1}(x(t), r(t)) \\
& h(x(t), r(t))=\frac{1}{2} u^{\top}(t) R(r(t)) u(t)
\end{aligned}
$$

with for all $\alpha \in \mathcal{S}, g_{0}(0, \alpha)=0$ and $g_{0}(x(t), \alpha)>0$ for all $x \neq 0$ and $g_{1}(0, \alpha)=$ 0 ; and $R(\alpha)$ is positive-definite matrix.

We will assume that the following:

$$
g_{0}(x(t), \alpha) \geq \rho(\alpha)\|x(t)\|^{2},
$$

hold for each $\alpha \in \mathcal{S}$.

Theorem 1 If the data of the previous nonlinear system is sufficiently smooth, therefore the optimality conditions of the nominal problem are given by:

$$
\begin{aligned}
\min _{u(t)}\left[\left(\mathcal{A}_{u} v\right)(x(t), r(t))+\right. & \left.g_{0}(x(t), r(t))+g_{1}(x(t), r(t))+\frac{1}{2} u^{\top}(t) R(r(t)) u(t)\right] \\
& =0
\end{aligned}
$$

where $\left(\mathcal{A}_{u} v\right)(x(t), r(t))$ is defined as follows:

$$
\begin{aligned}
\left(\mathcal{A}_{u} v\right)(x(t), r(t))= & v_{x}^{\top}(x(t), r(t))[A(x(t), r(t))+B(x(t), r(t)) u(t)] \\
& +\sum_{\beta \in \mathcal{S}} q_{\alpha \beta} v(x(t), \beta)
\end{aligned}
$$

The corresponding optimal control is given by:

$$
k(x(t), r(t))=-R^{-1}(r(t)) B^{\top}(r(t)) v_{x}^{\top}(x(t), r(t))
$$

Proof: The proof of this theorem can be adapted from the one in Boukas (1993).

\section{ROBUST STABILIZATION}

Let us now return to the optimization problem we formulated in section 2 in which we have an additive nonlinear state dependent disturbance $d(x(t), r(t))$ to the changes in the control gain which in turn are assumed to be also nonlinear 
uncertainties. Our goal in this section is to design a stabilizing control law for the corresponding uncertain system (2.1)-(2.2).

Theorem 2 If for every $\alpha \in \mathcal{S}$, there exist a positive number $\gamma(\alpha)$ such that the following conditions

$$
\begin{aligned}
& 2 p^{\top}(u(t)) R(\alpha) u(t)-[1+\gamma(\alpha)] u^{\top}(t) R(\alpha) u(t) \geq 0 \\
& 2 \gamma(\alpha) g_{1}(x(t), \alpha)-d^{\top}(x(t), \alpha) R(\alpha) d(x(t), \alpha) \geq 0
\end{aligned}
$$

hold. Then the optimal control law given by Eq. (4.6) is robustly stabilizes the uncertain system (2.1)-(2.2).

Proof: Let the value function be the candidate Lyapunov function. The infenitesimal operator of this function is given by:

$$
\begin{aligned}
\tilde{\mathcal{A}} v(x, r(t))= & v_{x}^{\top}(x, r(t))[A(x(t), \alpha)+B(x(t), r(t)) d(x(t), r(t)) \\
& +B(x(t), r(t)) p(k(x(t), r(t))]+\sum_{\beta \in \mathcal{S}} q_{\alpha \beta} v(x(t), \beta)
\end{aligned}
$$

After some calculation, using the conditions above, we can prove that

$$
\tilde{\mathcal{A}} v(x, r(t)) \leq-\rho\|x(t)\|^{2}
$$

where $\rho>0$.

The rest of the proof can be done as in Boukas and Yang (1997).

Remark The conditions (5.1)-(5.2) are conservative and the design approach requires the resolution of the optimization problem of the nominal nonlinear with Markovian jumping parameters.

The next theorem gives a less conservative conditions.

Theorem 3 If the condition (5.1) is satisfied and in some neighborhood $\mathcal{X}$ of $x=0$, for $x \neq 0$ the following:

$$
\begin{aligned}
2 k^{\top}(x(t), \alpha) R(\alpha) d(x(t), \alpha) & +\gamma(\alpha) k^{\top}(x(t), \alpha) R(\alpha) k(x(t), \alpha) \\
& +2 g_{1}(x(t), \alpha) \geq 0, \forall \alpha \in \mathcal{S}
\end{aligned}
$$

holds. Then $x=0$ is asymptotically stochatically stable equilibrium point of system (2.1)-(2.2) under the control law given by (4.6).

Proof: The proof of this theorem follows the same steps of the one of Theorem 2. The detail is omitted.

Remark Notice that the design of the control law requires the resolution on the optimization problem of the nonlinear nominal system with Markovian jumping parameters which is in general not an easy task. A design procedure that overcome this will be preferable.

Theorem 4 If the condition (5.1) is satisfied and that there exist a positive definite function $v(x(t), r(t))$ and positive number $\gamma_{1}(r(t))$ and $\gamma_{2}(r(t))$, such that in a neighborhood $\mathcal{X}$ of $x=0$, for $x \neq 0$ the following:

$$
2 \gamma_{2}(r(t))\left(1-\gamma_{1}(r(t))\left[-v_{x}^{\top}(x(t), \alpha) A(x(t), r(t))\right.\right.
$$




$$
\begin{aligned}
& -d^{\top}(x(t), r(t)) R(r(t)) d(x(t), r(t)) \\
& +\frac{1}{2} v_{x}^{\top}(x(t), r(t)) B(x(t), r(t)) R^{-1}(r(t)) B^{\top}(x(t), r(t)) v_{x}^{\top}(x(t), r(t)] \\
& \geq 0
\end{aligned}
$$

holds. Then under the feedback control law (4.6), $x=0$ is asymptotically stochatically stable equilibrium point of system (2.1)-(2.2).

Proof: The proof of this theorem follows the same steps of the one of Theorem 2. The detail is omitted.

\section{NUMERICAL EXAMPLES}

Consider a production system consisting of one machine producing one item. Let the Markov process $r(t)$ has two modes, i.e., $\mathcal{S}=\{1,2\}$, and let its dynamics be described by the following transition matrix:

$$
Q=\left[\begin{array}{cc}
-0.1 & 0.1 \\
0.5 & -0.5
\end{array}\right]
$$

- $\quad$ mode 1 :

$$
\begin{aligned}
& \dot{x}_{1}(t)=-x_{1}(t) \\
& \dot{x}_{2}(t)=-x_{2}(t)+u-\frac{a_{1}(t) \sin x_{1}(t)}{l_{1}}
\end{aligned}
$$

where $l_{1}$ is a positive constant and $a_{1}(t)$ is the uncertainty.

- $\quad$ mode 2 :

$$
\begin{aligned}
& \dot{x}_{1}(t)=-x_{1}(t) \\
& \dot{x}_{2}(t)=-x_{2}(t)+u-\frac{a_{2}(t) \sin x_{1}(t)}{l_{2}}
\end{aligned}
$$

where $l_{2}$ is a positive constant and $a_{2}(t)$ is the uncertainty.

Let

$$
R(r(t))=\left[\begin{array}{ll}
1 & 0 \\
0 & 1
\end{array}\right]
$$

for all $r(t) \in \mathcal{S}$. In this example, for both mode 1 and mode 2 ,

$$
\begin{aligned}
A & =\left[\begin{array}{c}
-x_{1}(t) \\
-x_{2}(t)
\end{array}\right] \\
B & =\left[\begin{array}{ll}
0 & 0 \\
0 & 1
\end{array}\right] \\
p(u(t)) & =\left[\begin{array}{c}
0 \\
u(t)
\end{array}\right]
\end{aligned}
$$


for mode 1 ,

$$
d(x(t))=\left[\begin{array}{c}
0 \\
-\frac{a_{1}(t) \sin x_{1}(t)}{l_{1}}
\end{array}\right]
$$

for mode 2 ,

$$
d(x(t))=\left[\begin{array}{c}
0 \\
-\frac{a_{2}(t) \sin x_{1}(t)}{l_{2}}
\end{array}\right]
$$

Assume that $g(x(t), r(t))=g_{0}(x(t), r(t))+g_{1}(x(t), r(t))$ satisfies that $g_{0}(x(t), r(t)) \geq$ $\frac{1}{2}\|x(t)\|^{2}$ for all $r(t)$, and $g_{1}(x(t), r(t)) \geq L>0$. If we assume that the uncertainties satisfy the following condition:

$$
\frac{\left|a_{1}(t) a_{2}(t)\right|}{l_{1} l_{2}} \leq L
$$

Then conditions (5.1)-(5.2) of Theorem 2 are satisfied, and the system is robustly stabilizable.

\section{References}

[1] Boukas, E. K., (1993). "Control of systems with controlled jump Markov disturbances," Control Theory and Advanced Technology, 9(2), 577-595.

[2] Boukas, E. K., (1995). "Robust stability of linear piecewise deterministic systems under matching conditions," Control Theory and Advanced Technology, 10(4), 1541-1549.

[3] Boukas, E. K. and H. Yang, (1997). "Robust stabilization by dynamic combined state and output feedback compensator for nonlinear systems with jumps," Journal of Optimization Theory and Applications, 92(1), 63-75.

[4] Gershwin, S. B. (1994) Manufacturing Systems Engineering, Prentice Hall.

[5] Ji, Y. and H. J. Chizeck, (1990). "Controllability, stabilizability, and continuous-time Markovian jump linear quadratic control," IEEE Trans. Automat. Control, 35(7), 777-788.

[6] Mariton, M., (1990). "Jump linear systems in Automatic control," Marcel Dekker, New York.

[7] Rishel, R., (1975). "Control of systems with jump Markov disturbances," IEEE Trans. Automat. Control, 24(4), 241-244.

[8] Sethi, S. P. and Q. Zhang, (1994). Hierarchical decision making in stochastic manufacturing systems. Birkhauser, Boston-Basel, Berlin. 